\title{
Approximation Solution of Linear Fredholm-Stieltjes Integral Equations of Second Kind Using Generalized Simpson's Quadrature Method
}

\author{
Isa Muslu' ${ }^{1}$, AvytAsanov ${ }^{2}$, Sedat Yanik ${ }^{3}$ \\ ${ }^{1}$ Bishkek (Kyrgyzstan), ${ }^{2}$ Bishkek (Kyrgyzstan), ${ }^{3}$ Bishkek (Kyrgyzstan)
}

Abstract: TheLinear Fredholm-Stieltjes Integral Equation of the Second Kind (LFSIESK) is of wide spread use in many fields of engineering and applied mathematics. The quadrature methods are remarkable among the variety of numerical solutions to this equation. The aim of this paper is to find an approximation solution of the LFSIESK using the quadrature method the Generalized Simpson's Method (GSM). Numerical example is presented at the end to show the efficiency and accuracy of the presented work. The software Maple 18 is used for the computations.

Keywords: Fredholm integral equation, generalized Simpson's rule, Stieltjes integrals, linear integral equation, Quadrature method.

\section{INTRODUCTION}

Integral Equations (IE) are frequently being used in different areas of applied mathematics, physics, and engineering etc. [1-6].The LFSIESK is one of the most practical ones[7-9]. A number of numerical solutions have already been proposed to this equation [10-17]. Nevertheless, an efficient low-cost solution to this equation has remained a scientific inquiry. In particular, the modification made to the quadrature method is still of high complexity.

Consider the following LFSIESK,

$u(x)=f(x)+\lambda \int_{a}^{b} k(x, t) u(t) d \alpha(t)$

Where $a \leq x \leq b, a \leq t \leq b, K(x, t)$-kernel of the function, $u(x)$-unknown function, $f(x)$-givenfunction, $\alpha(t)$-strictly increasing function and $\lambda \in R$.If $\alpha(t)=t$,then it is LFSIESK and the studies about its approximation solutions using quadrature methods can be found in [1-2].

In this article it was taken if $\alpha(t) \neq t$.The Approximation quadrature method, Generalized Simpson Method to compute Stieltjes integrals are given in [14],[15], and[16], respectively. These methods were used to find approximate solution of LFSIESK in (1).

\section{NUMERICAL SOLUTION OF LFSIESK USING THE GTM}

$I=\int_{a}^{b} f(x) d g(x), h=\frac{b-a}{n}, x_{i}=a+i h, i=0,1,2, \ldots, 2 n, n \in N$

where $f(x)$ is a given continuous function on $[a, b]$ and $g(x)$ is a given function ofbounded variation on $[a, b]$. It is known $[16,205 \mathrm{p}$.] , that the function $g(x)$ presented in the form

$$
g(x)=\phi(x)-\psi(x), x \in[a, b]
$$

\footnotetext{
${ }^{1}$ Corresponding Author: isa.muslu@iaau.edu.kg
} 
where $\varphi(x)$ and $\psi(x)$ are the known increasing functions, on $[a, b]$.

Solving the LFIESK using the GSM, we get

$$
\begin{aligned}
& A_{n}=\frac{1}{6} \sum_{i=0}^{n-1}\left\{\left[\phi\left(x_{2 i+2}\right)-\phi\left(x_{2 i}\right)\right]\left[f\left(x_{2 i}\right)+4 f\left(x_{2 i+1}^{*}\right)+f\left(x_{2 i+2}\right)\right]-\left[\psi\left(x_{2 i+2}\right)-\psi\left(x_{2 i}\right)\right]\left[f\left(x_{2 i}\right)+4 f\left(x^{* *}{ }_{2 i+1}\right)+f\left(x_{2 i+2}\right)\right]\right\} \\
& \phi\left(x_{2 i+1}^{*}\right)=\frac{1}{2}\left[\phi\left(x_{2 i}\right)+\phi\left(x_{2 i+2}\right)\right], \psi\left(x * *_{2 i+1}\right)=\frac{1}{2}\left[\psi\left(x_{2 i}\right)+\psi\left(x_{2 i+2}\right)\right] \\
& u(x)=\int_{a}^{b} K(x, y) u(y) d g(y)+f(x),=\underbrace{\int_{a}^{b} K(x, y) u(y) d \phi(y)}_{I}-\underbrace{\int_{a}^{b} K(x, y) u(y) d \psi(y)}_{I I}+f(x), x \in[a, b] \\
& I=\int_{a}^{b} K(x, y) u(y) d \phi(y) \approx \frac{1}{6} \sum_{i=0}^{n-1}\left[K\left(x, x_{2 i}\right) u\left(x_{2 i}\right)+4 K\left(x, x_{2 i+1}^{*}\right) u\left(x_{2 i+1}^{*}\right)+K\left(x, x_{2 i+2}\right) u\left(x_{2 i+2}\right)\right] \cdot\left[\phi\left(x_{2 i+2}\right)-\phi\left(x_{2 i}\right)\right] \\
& =\frac{1}{6}\left\{\left[K\left(x, x_{0}\right) u\left(x_{0}\right)+4 K\left(x, x_{1}^{*}\right) u\left(x_{1}^{*}\right)+K\left(x, x_{2}\right) u\left(x_{2}\right)\right] \cdot\left[\phi\left(x_{2}\right)-\phi\left(x_{0}\right)\right]+\right. \\
& +\left[K\left(x, x_{2}\right) u\left(x_{2}\right)+4 K\left(x, x_{3}^{*}\right) u\left(x_{3}^{*}\right)+K\left(x, x_{4}\right) u\left(x_{4}\right)\right] \cdot\left[\phi\left(x_{4}\right)-\phi\left(x_{2}\right)\right]+\ldots .+ \\
& \left.+\left[K\left(x, x_{2 n-2}\right) u\left(x_{2 n-2}\right)+4 K\left(x, x_{2 n-1}^{*}\right) u\left(x_{2 n-1}^{*}\right)+K\left(x, x_{2 n}\right) u\left(x_{2 n}\right)\right] \cdot\left[\phi\left(x_{2 n}\right)-\phi\left(x_{2 n-2}\right)\right]\right\}, x \in[a, b]
\end{aligned}
$$

where

$$
\begin{aligned}
x_{1}^{*} & =\phi^{-1}\left(\frac{\phi\left(x_{2}\right)+\phi\left(x_{0}\right)}{2}\right), x_{3}^{*}=\phi^{-1}\left(\frac{\phi\left(x_{4}\right)+\phi\left(x_{2}\right)}{2}\right), x_{2 n-1}^{*}=\phi^{-1}\left(\frac{\phi\left(x_{2 n}\right)+\phi\left(x_{2 n-2}\right)}{2}\right) \\
I I & =\int_{a}^{b} K(x, y) u(y) d \psi(y) \approx \frac{1}{6} \sum_{i=0}^{n-1}\left[K\left(x, x_{2 i}\right) u\left(x_{2 i}\right)+4 K\left(x, x^{* *}{ }_{2 i+1}\right) u\left(x^{* *}{ }_{2 i+1}\right)+K\left(x, x_{2 i+2}\right) u\left(x_{2 i+2}\right)\right] \cdot\left[\psi\left(x_{2 i+2}\right)-\psi\left(x_{2 i}\right)\right] \\
& =\frac{1}{6}\left\{\left[K\left(x, x_{0}\right) u\left(x_{0}\right)+4 K\left(x, x^{* *}\right) u\left(x^{* *}{ }_{1}\right)+K\left(x, x_{2}\right) u\left(x_{2}\right)\right] \cdot\left[\psi\left(x_{2}\right)-\psi\left(x_{0}\right)\right]+\right. \\
& +\left[K\left(x, x_{2}\right) u\left(x_{2}\right)+4 K\left(x, x_{3}^{* *}\right) u\left(x_{3}^{* *}\right)+K\left(x, x_{4}\right) u\left(x_{4}\right)\right] \cdot\left[\psi\left(x_{4}\right)-\psi\left(x_{2}\right)\right]+\ldots+ \\
& \left.+\left[K\left(x, x_{2 n-2}\right) u\left(x_{2 n-2}\right)+4 K\left(x, x_{2 n-1}^{* *}\right) u\left(x_{2 n-1}^{* *}\right)+K\left(x, x_{2 n}\right) u\left(x_{2 n}\right)\right] \cdot\left[\psi\left(x_{2 n}\right)-\psi\left(x_{2 n-2}\right)\right]\right\}
\end{aligned}
$$

Where

$$
\begin{aligned}
x_{1}^{* * *} & =\psi^{-1}\left(\frac{\psi\left(x_{2}\right)+\psi\left(x_{0}\right)}{2}\right), x_{3}^{* *}=\psi^{-1}\left(\frac{\psi\left(x_{4}\right)+\psi\left(x_{2}\right)}{2}\right), \ldots, x_{2 n-1} * *=\psi^{-1}\left(\frac{\psi\left(x_{2 n}\right)+\psi\left(x_{2 n-2}\right)}{2}\right) \\
u(x) & \approx \frac{1}{6}\left\{\left[K\left(x, x_{0}\right) u\left(x_{0}\right)+4 K\left(x, x_{1}^{*}\right) u\left(x_{1}^{*}\right)+K\left(x, x_{2}\right) u\left(x_{2}\right)\right] \varphi\left(x_{2}\right)-\varphi\left(x_{0}\right)\right]+ \\
& \left.+\left[K\left(x, x_{2}\right) u\left(x_{2}\right)+4 K\left(x, x_{3}^{*}\right) u\left(x_{3}^{*}\right)+K\left(x, x_{4}\right) u\left(x_{4}\right)\right] \varphi\left(x_{4}\right)-\varphi\left(x_{2}\right)\right]+\ldots \ldots+ \\
& \left.+\left[K\left(x, x_{2 n-2}\right) u\left(x_{2 n-2}\right)+4 K\left(x, x_{2 n-1}^{*}\right) u\left(x_{2 n-1}^{*}\right)+K\left(x, x_{2 n}\right) u\left(x_{2 n}\right)\right] \varphi\left(x_{2 n}\right)-\varphi\left(x_{2 n-2}\right)\right]- \\
& -\left[K\left(x, x_{0}\right) u\left(x_{0}\right)+4 K\left(x, x_{1}^{* *}\right) u\left(x_{1}^{* *}\right)+K\left(x, x_{2}\right) u\left(x_{2}\right)\right]\left[\psi\left(x_{2}\right)-\psi\left(x_{0}\right)\right]- \\
& \left.-\left[K\left(x, x_{2}\right) u\left(x_{2}\right)+4 K\left(x, x_{3}^{* *}\right) u\left(x_{3}^{* *}\right)+K\left(x, x_{4}\right) u\left(x_{4}\right)\right] \psi\left(x_{4}\right)-\psi\left(x_{2}\right)\right]-\ldots \ldots- \\
& \left.-\left[K\left(x, x_{2 n-2}\right) u\left(x_{2 n-2}\right)+4 K\left(x, x_{2 n-1}{ }^{* *}\right) u\left(x_{2 n-1}^{* *}\right)+K\left(x, x_{2 n}\right) u\left(x_{2 n}\right)\right]\left[\psi\left(x_{2 n}\right)-\psi\left(x_{2 n-2}\right)\right]\right\}+f(x)
\end{aligned}
$$


$\approx \frac{1}{6}\left\{\left[K\left(x, x_{0}\right)\left[\varphi\left(x_{2}\right)-\varphi\left(x_{0}\right)\right] u\left(x_{0}\right)+4 K\left(x, x_{1}^{*}\right)\left[\varphi\left(x_{2}\right)-\varphi\left(x_{0}\right)\right) u\left(x_{1}^{*}\right)+K\left(x, x_{2}\right)\left[\varphi\left(x_{2}\right)-\varphi\left(x_{0}\right)\right] u\left(x_{2}\right)\right]+\right.$ $+\left[K\left(x, x_{2}\right)\left[\varphi\left(x_{4}\right)-\varphi\left(x_{2}\right)\right] u\left(x_{2}\right)+4 K\left(x, x_{3}^{*}\right)\left[\varphi\left(x_{4}\right)-\varphi\left(x_{2}\right)\right] u\left(x_{3}^{*}\right)+K\left(x, x_{4}\right)\left[\varphi\left(x_{4}\right)-\varphi\left(x_{2}\right)\right] u\left(x_{4}\right)\right]+\ldots+$ $+\left[K\left(x, x_{2 n-2}\right)\left[\varphi\left(x_{2 n}\right)-\varphi\left(x_{2 n-2}\right)\right] u\left(x_{2 n-2}\right)+4 K\left(x, x_{2 n-1}\right)\left[\varphi\left(x_{2 n}\right)-\varphi\left(x_{2 n-2}\right)\right] u\left(x_{2 n-1}\right)+K\left(x, x_{2 n}\right)\left[\varphi\left(x_{2 n}\right)-\varphi\left(x_{2 n-2}\right)\right] u\left(x_{2 n}\right)\right]-$ $-\left[K\left(x, x_{0}\right)\left[\psi\left(x_{2}\right)-\psi\left(x_{0}\right)\right] u\left(x_{0}\right)+4 K\left(x, x_{1}^{* * *}\right)\left[\psi\left(x_{2}\right)-\psi\left(x_{0}\right)\right] u\left(x_{1}^{* *}\right)+K\left(x, x_{2}\right)\left[\psi\left(x_{2}\right)-\psi\left(x_{0}\right)\right] u\left(x_{2}\right)\right]-$ $-\left[K\left(x, x_{2}\right)\left[\psi\left(x_{4}\right)-\psi\left(x_{2}\right)\right] u\left(x_{2}\right)+4 K\left(x, x_{3}^{* \prime \prime}\right)\left[\psi\left(x_{4}\right)-\psi\left(x_{2}\right)\right] u\left(x_{3}^{* *}\right)+K\left(x, x_{4}\right)\left[\psi\left(x_{4}\right)-\psi\left(x_{2}\right)\right] u\left(x_{4}\right)\right]-$ $\left.-\left[K\left(x, x_{2 n-2}\right)\left[\psi\left(x_{2 n}\right)-\psi\left(x_{2 n-2}\right)\right] u\left(x_{2 n-2}\right)+4 K\left(x, x_{2 n-1}{ }^{4 \prime}\right)\left[\psi\left(x_{2 n}\right)-\psi\left(x_{2 n-2}\right)\right] u\left(x_{2 n-1}{ }^{*}\right)+K\left(x, x_{2 n}\right)\left[\psi\left(x_{2 n}\right)-\psi\left(x_{2 n-2}\right)\right] u\left(x_{2 n}\right)\right]\right\}+$ $+f(x)$

$\approx \frac{1}{6}\left\{K\left(x, x_{0}\right)\left[\left[\varphi\left(x_{2}\right)-\varphi\left(x_{0}\right)\right]-\left[\psi\left(x_{2}\right)-\psi\left(x_{0}\right)\right]\right]\right\} u\left(x_{0}\right)+\frac{4}{6} K\left(x, x_{1}^{*}\right)\left[\varphi\left(x_{2}\right)-\varphi\left(x_{0}\right)\right) u\left(x_{1} *\right)+$ $+\frac{1}{6}\left\{K\left(x, x_{2}\right)\left[\left[\varphi\left(x_{2}\right)-\varphi\left(x_{0}\right)\right]+\left[\varphi\left(x_{4}\right)-\varphi\left(x_{2}\right)\right]-\left[\psi\left(x_{2}\right)-\psi\left(x_{0}\right)\right]-\left[\psi\left(x_{4}\right)-\psi\left(x_{2}\right)\right]\right]\right\} u\left(x_{2}\right)+$ $\left.+\frac{4}{6} K\left(x, x_{3} *\right)\left[\varphi\left(x_{4}\right)-\varphi\left(x_{2}\right)\right] u\left(x_{3} *\right)+\frac{1}{6}\left\{K\left(x, x_{4}\right)\left[\varphi\left(x_{4}\right)-\varphi\left(x_{2}\right)\right]+\left[\varphi\left(x_{6}\right)-\varphi\left(x_{4}\right)\right]-\left[\psi\left(x_{4}\right)-\psi\left(x_{2}\right)\right]-\left[\psi\left(x_{6}\right)-\psi\left(x_{4}\right)\right]\right]\right\}\left(x_{4}\right)+\ldots .+$ $+\frac{4}{6} K\left(x, x_{2 n-1} *\left[\varphi\left(x_{2 n}\right)-\varphi\left(x_{2 n-2}\right)\right] u\left(x_{2 n-1} *\right)+\frac{1}{6}\left\{K\left(x, x_{2 n}\right)\left[\varphi\left(x_{2 n}\right)-\varphi\left(x_{2 n-2}\right)\right]-\left[\psi\left(x_{2 n}\right)-\psi\left(x_{2 n-2}\right)\right]\right\} u\left(x_{2 n}\right)+\right.$ $+\frac{4}{6} K\left(x, x_{1} * *\right)\left[\psi\left(x_{2}\right)-\psi\left(x_{0}\right)\right] u\left(x_{1} * *\right)+\frac{4}{6} K\left(x, x_{3} * *\right)\left[\psi\left(x_{4}\right)-\psi\left(x_{2}\right)\right] u\left(x_{3} * *\right)+\ldots+\frac{4}{6} K\left(x, x_{2 n-1} * *\right)\left[\psi\left(x_{2 n}\right)-\psi\left(x_{2 n-2}\right)\right] u\left(x_{2 n-1} * *\right)+$ $+f(x)$

$$
\begin{aligned}
u(x) & \approx A_{0}(x) u\left(x_{0}\right)+A_{1}(x) u\left(x_{1} *\right)+A_{2}(x) u\left(x_{2}\right)+\ldots+A_{2 n-1}(x) u\left(x_{2 n-1} *\right)+A_{2 n}(x) u\left(x_{2 n}\right)+ \\
& +B_{1}(x) u\left(x_{1} * *\right)+B_{2}(x) u\left(x_{3} * *\right)+\ldots+B_{n}(x) u\left(x_{2 n-1} *\right)+f(x)
\end{aligned}
$$

Where,

$$
\begin{aligned}
& A_{0}(x)=\frac{1}{6}\left\{K\left(x, x_{0}\right)\left[\left[\phi\left(x_{2}\right)-\phi\left(x_{0}\right)\right]-\left[\psi\left(x_{2}\right)-\psi\left(x_{0}\right)\right]\right]\right\}, A_{1}(x)=\frac{2}{3} K\left(x, x_{1}^{*}\right)\left[\phi\left(x_{2}\right)-\phi\left(x_{0}\right)\right] \\
& A_{2}(x)=\frac{1}{6}\left\{K\left(x, x_{2}\right)\left[\left[\phi\left(x_{2}\right)-\phi\left(x_{0}\right)\right]+\left[\phi\left(x_{4}\right)-\phi\left(x_{2}\right)\right]-\left[\psi\left(x_{2}\right)-\psi\left(x_{0}\right)\right]-\left[\psi\left(x_{4}\right)-\psi\left(x_{2}\right)\right]\right]\right\} \ldots \\
& A_{2 n-1}(x)=\frac{2}{3} K\left(x, x_{2 n-1} *\left[\phi\left(x_{2 n}\right)-\phi\left(x_{2 n-2}\right)\right], A_{2 n}(x)=\frac{1}{6}\left\{K\left(x, x_{2 n}\right)\left[\left[\phi\left(x_{2 n}\right)-\phi\left(x_{2 n-2}\right)\right]-\left[\psi\left(x_{2 n}\right)-\psi\left(x_{2 n-2}\right)\right]\right]\right\}\right. \\
& B_{1}(x)=\frac{2}{3} K\left(x, x_{1} * *\right)\left[\psi\left(x_{2}\right)-\psi\left(x_{0}\right)\right], B_{2}(x)=\frac{2}{3} K\left(x, x_{3} * *\right)\left[\psi\left(x_{4}\right)-\psi\left(x_{2}\right)\right], \ldots, B_{n}(x)=\frac{2}{3} K\left(x, x_{2 n-1} * *\right)\left[\psi\left(x_{2 n}\right)-\psi\left(x_{2 n-2}\right)\right] \\
& u\left(x_{0}\right) \quad \approx A_{0}\left(x_{0}\right) u\left(x_{0}\right)+A_{1}\left(x_{0}\right) u\left(x_{1}^{*}\right)+A_{2}\left(x_{0}\right) u\left(x_{2}\right)+\ldots+A_{2 n-1}\left(x_{0}\right) u\left(x_{2 n-1}^{*}\right)+A_{2 n}\left(x_{0}\right) u\left(x_{2 n}\right)+ \\
& +B_{1}\left(x_{0}\right) u\left(x_{1}^{* * *}\right)+B_{2}\left(x_{0}\right) u\left(x_{3}^{* * *}\right)+B_{3}\left(x_{0}\right) u\left(x_{5}^{* *}\right)+\ldots+B_{n}\left(x_{0}\right) u\left(x_{2 n-1}{ }^{* * *}\right)+f\left(x_{0}\right) \\
& u\left(x_{1}^{*}\right) \quad \approx A_{0}\left(x_{1}^{*}\right) u\left(x_{0}\right)+A_{1}\left(x_{1}^{*}\right) u\left(x_{1}^{*}\right)+A_{2}\left(x_{1}^{*}\right) u\left(x_{2}\right)+\ldots+A_{2 n-1}\left(x_{1}^{*}\right) u\left(x_{2 n-1}{ }^{*}\right)+A_{2 n}\left(x_{1}^{*}\right) u\left(x_{2 n}\right)+ \\
& +B_{1}\left(x_{1}^{*}\right) u\left(x_{1}^{* *}\right)+B_{2}\left(x_{1}^{*}\right) u\left(x_{3}^{* * *}\right)+B_{3}\left(x_{1}^{*}\right) u\left(x_{5}^{* * *}\right)+\ldots+B_{n}\left(x_{1}^{*}\right) u\left(x_{2 n-1}^{* * *}\right)+f\left(x_{1}^{*}\right) \\
& u\left(x_{2}\right) \quad \approx A_{0}\left(x_{2}\right) u\left(x_{0}\right)+A_{1}\left(x_{2}\right) u\left(x_{1}^{*}\right)+A_{2}\left(x_{2}\right) u\left(x_{2}\right)+\ldots+A_{2 n-1}\left(x_{2}\right) u\left(x_{2 n-1}{ }^{*}\right)+A_{2 n}\left(x_{2}\right) u\left(x_{2 n}\right)+ \\
& +B_{1}\left(x_{2}\right) u\left(x_{1}^{* *}\right)+B_{2}\left(x_{2}\right) u\left(x_{3}^{* * *}\right)+B_{3}\left(x_{2}\right) u\left(x_{5}^{* * *}\right)+\ldots+B_{n}\left(x_{2}\right) u\left(x_{2 n-1}^{* *}\right)+f\left(x_{2}\right) \ldots \\
& u\left(x_{2 n-1}{ }^{*}\right) \quad \approx A_{0}\left(x_{2 n-1}{ }^{*}\right) u\left(x_{0}\right)+A_{1}\left(x_{2 n-1}{ }^{*}\right) u\left(x_{1}{ }^{*}\right)+A_{2}\left(x_{2 n-1}{ }^{*}\right) u\left(x_{2}\right)+\ldots+A_{2 n-1}\left(x_{2 n-1}{ }^{*}\right) u\left(x_{2 n-1}{ }^{*}\right)+A_{2 n}\left(x_{2 n-1}{ }^{*}\right) u\left(x_{2 n}\right)+ \\
& +B_{1}\left(x_{2 n-1}{ }^{*}\right) u\left(x_{1}^{* * *}\right)+B_{2}\left(x_{2 n-1}{ }^{*}\right) u\left(x_{3}^{* * *}\right)+B_{3}\left(x_{2 n-1}{ }^{*}\right) u\left(x_{5}^{* * *}\right)+\ldots+B_{n}\left(x_{2 n-1}{ }^{*}\right) u\left(x_{2 n-1}{ }^{* * *}\right)+f\left(x_{2 n-1}{ }^{*}\right)
\end{aligned}
$$




$$
\begin{aligned}
& u\left(x_{2 n}\right) \quad \approx A_{0}\left(x_{2 n}\right) u\left(x_{0}\right)+A_{1}\left(x_{2 n}\right) u\left(x_{1}^{*}\right)+A_{2}\left(x_{2 n}\right) u\left(x_{2}\right)+\ldots+A_{2 n-1}\left(x_{2 n}\right) u\left(x_{2 n-1}^{*}\right)+A_{2 n}\left(x_{2 n}\right) u\left(x_{2 n}\right)+ \\
& +B_{1}\left(x_{2 n}\right) u\left(x_{1}^{* *}\right)+B_{2}\left(x_{2 n}\right) u\left(x_{3}^{* *}\right)+B_{3}\left(x_{2 n}\right) u\left(x_{5}^{* *}\right)+\ldots+B_{n}\left(x_{2 n}\right) u\left(x_{2 n-1}^{* *}\right)+f\left(x_{2 n}\right) \\
& u\left(x_{1}^{* * *}\right) \quad \approx A_{0}\left(x_{1}^{* *}\right) u\left(x_{0}\right)+A_{1}\left(x_{1}^{* *}\right) u\left(x_{1}^{*}\right)+A_{2}\left(x_{1}^{* *}\right) u\left(x_{2}\right)+\ldots+A_{2 n-1}\left(x_{1}^{* * *}\right) u\left(x_{2 n-1}^{* * *}\right)+A_{2 n}\left(x_{1}^{* *}\right) u\left(x_{2 n}\right)+ \\
& +B_{1}\left(x_{1}^{* *}\right) u\left(x_{1}^{* *}\right)+B_{2}\left(x_{1}^{* *}\right) u\left(x_{3}^{* *}\right)+B_{3}\left(x_{1}^{* *}\right) u\left(x_{5}^{* *}\right)+\ldots+B_{n}\left(x_{1}^{* *}\right) u\left(x_{2 n-1}^{* *}\right)+f\left(x_{1}^{* *}\right) \\
& u\left(x_{3}^{* *}\right) \quad \approx A_{0}\left(x_{3}^{* *}\right) u\left(x_{0}\right)+A_{1}\left(x_{3}^{* *}\right) u\left(x_{1}^{*}\right)+A_{2}\left(x_{3}^{* *}\right) u\left(x_{2}\right)+\ldots+A_{2 n-1}\left(x_{3}^{* *}\right) u\left(x_{2 n-1}^{* *}\right)+A_{2 n}\left(x_{3}^{* *}\right) u\left(x_{2 n}\right)+ \\
& +B_{1}\left(x_{3}^{* *}\right) u\left(x_{1}^{* *}\right)+B_{2}\left(x_{3}^{* *}\right) u\left(x_{3}^{* *}\right)+B_{3}\left(x_{3}^{* *}\right) u\left(x_{5}^{* *}\right)+\ldots+B_{n}\left(x_{3}^{* *}\right) u\left(x_{2 n-1}{ }^{* *}\right)+f\left(x_{3}^{* *}\right) \\
& u_{0}=A_{0}\left(x_{0}\right) u_{0}+A_{1}(x) u_{1}^{*}+A_{2}\left(x_{0}\right) u_{2}+\ldots+A_{2 n-1}\left(x_{0}\right) u_{2 n-1}{ }^{*}+A_{2 n}\left(x_{0}\right) u_{2 n}+ \\
& +B_{1}\left(x_{0}\right) u_{1}^{* *}+B_{2}\left(x_{0}\right) u_{3}^{* *}+B_{3}\left(x_{0}\right) u_{5}^{* *}+\ldots+B_{n}\left(x_{0}\right) u_{2 n-1}{ }^{* *}+f\left(x_{0}\right) \\
& u_{1}^{*}=A_{0}\left(x_{1}^{*}\right) u_{0}+A_{1}\left(x_{1}^{*}\right) u_{1}^{*}+A_{2}\left(x_{1}^{*}\right) u_{2}+\ldots+A_{2 n-1}\left(x_{1}^{*}\right) u_{2 n-1}^{*}+A_{2 n}\left(x_{1}^{*}\right) u_{2 n}+ \\
& +B_{1}\left(x_{1}^{*}\right) u_{1}^{* *}+B_{2}\left(x_{1}^{*}\right) u_{3}^{* *}+B_{3}\left(x_{1}^{*}\right) u_{5}^{* *}+\ldots+B_{n}\left(x_{1}^{*}\right) u_{2 n-1}^{* *}+f\left(x_{1}^{*}\right) \\
& u_{2}=A_{0}\left(x_{2}\right) u_{0}+A_{1}\left(x_{2}\right) u_{1}^{*}+A_{2}\left(x_{2}\right) u_{2}+\ldots+A_{2 n-1}\left(x_{2}\right) u_{2 n-1}{ }^{*}+A_{2 n}\left(x_{2}\right) u_{2 n}+ \\
& +B_{1}\left(x_{2}\right) u_{1}^{* *}+B_{2}\left(x_{2}\right) u_{3}^{* *}+B_{3}\left(x_{2}\right) u_{5}^{* *}+\ldots+B_{n}\left(x_{2}\right) u_{2 n-1}^{* *}+f\left(x_{2}\right) \ldots \\
& u_{2 n-1}^{*}=A_{0}\left(x_{2 n-1}^{*}\right) u_{0}+A_{1}\left(x_{2 n-1}^{*}\right) u_{1}^{*}+A_{2}\left(x_{2 n-1}^{*}\right) u_{2}+\ldots+A_{2 n-1}\left(x_{2 n-1}^{*}\right) u_{2 n-1}^{*}+A_{2 n}\left(x_{2 n-1}^{*}\right) u_{2 n}+ \\
& +B_{1}\left(x_{2 n-1}^{*}\right) u_{1}^{* *}+B_{2}\left(x_{2 n-1}^{*}\right) u_{3}^{* *}+B_{3}\left(x_{2 n-1}^{*}\right) u_{5}^{* *}+\ldots+B_{n}\left(x_{2 n-1}^{*}\right) u_{2 n-1}^{* *}+f\left(x_{2 n-1}^{*}\right) \\
& u_{2 n}=A_{0}\left(x_{2 n}\right) u_{0}+A_{1}\left(x_{2 n}\right) u_{1}^{*}+A_{2}\left(x_{2 n}\right) u_{2}+\ldots+A_{2 n-1}\left(x_{2 n}\right) u_{2 n-1}^{*}+A_{2 n}\left(x_{2 n}\right) u_{2 n}+ \\
& +B_{1}\left(x_{2 n}\right) u_{1}^{* *}+B_{2}\left(x_{2 n}\right) u_{3}^{* *}+B_{3}\left(x_{2 n}\right) u_{5}^{* *}+\ldots+B_{n}\left(x_{2 n}\right) u_{2 n-1}^{* *}+f\left(x_{2 n}\right) \\
& u_{1}^{* *}=A_{0}\left(x_{1}^{* *}\right) u_{0}+A_{1}\left(x_{1}^{* *}\right) u_{1}^{*}+A_{2}\left(x_{1}^{* *}\right) u_{2}+\ldots+A_{2 n-1}\left(x_{1}^{* *}\right) u_{2 n-1}^{* *}+A_{2 n}\left(x_{1}^{* *}\right) u_{2 n}+ \\
& +B_{1}\left(x_{1}^{* *}\right) u_{1}^{* *}+B_{2}\left(x_{1}^{* *}\right) u_{3}^{* *}+B_{3}\left(x_{1}^{* *}\right) u_{5}^{* *}+\ldots+B_{n}\left(x_{1}^{* *}\right) u_{2 n-1}^{* *}+f\left(x_{1}^{* *}\right) \\
& u_{3}^{* *}=A_{0}\left(x_{3}^{* *}\right) u_{0}+A_{1}\left(x_{3}^{* *}\right) u_{1}^{*}+A_{2}\left(x_{3}^{* *}\right) u_{2}+\ldots+A_{2 n-1}\left(x_{3}^{* *}\right) u_{2 n-1}^{* *}+A_{2 n}\left(x_{3}^{* *}\right) u_{2 n}+ \\
& +B_{1}\left(x_{3}^{* *}\right) u_{1}^{* *}+B_{2}\left(x_{3}^{* *}\right) u_{3}^{* *}+B_{3}\left(x_{3}^{* *}\right) u_{5}^{* *}+\ldots+B_{n}\left(x_{3}^{* *}\right) u_{2 n-1}^{* *}+f\left(x_{3}^{* *}\right) \ldots \\
& u_{2 n-1}{ }^{* *}=A_{0}\left(x_{2 n-1}^{* *}\right) u_{0}+A_{1}\left(x_{2 n-1}^{* *}\right) u_{1}^{*}+A_{2}\left(x_{2 n-1}^{* *}\right) u_{2}+\ldots+A_{2 n-1}\left(x_{2 n-1}^{* *}\right) u_{2 n-1}^{*}+A_{2 n}\left(x_{2 n-1}^{* *}\right) u_{2 n}+ \\
& +B_{1}\left(x_{2 n-1}^{* *}\right) u_{1}^{* * *}+B_{2}\left(x_{2 n-1}^{* *}\right) u_{3}^{* *}+B_{3}\left(x_{2 n-1}^{* *}\right) u_{5}^{* *}+\ldots+B_{n}\left(x_{2 n-1}^{* *}\right) u_{2 n-1}^{* *}+f\left(x_{2 n-1}^{* *}\right)
\end{aligned}
$$

Taking every term to the leftside for each equation (making them homogeneous) we get the following system of equations $(*)$

$$
\begin{aligned}
& \left(1-A_{0}\left(x_{0}\right)\right) u_{0}-A_{1}(x) u_{1}^{*}-A_{2}\left(x_{0}\right) u_{2}-\ldots-A_{2 n-1}\left(x_{0}\right) u_{2 n-1}{ }^{*}-A_{2 n}\left(x_{0}\right) u_{2 n}- \\
& -B_{1}\left(x_{0}\right) u_{1}^{* *}-B_{2}\left(x_{0}\right) u_{3}^{* *}-B_{3}\left(x_{0}\right) u_{5}^{* *}-\ldots-B_{n}\left(x_{0}\right) u_{2 n-1}^{* *}=f\left(x_{0}\right) \\
& -A_{0}\left(x_{1}^{*}\right) u_{0}+\left(1-A_{1}\left(x_{1}^{*}\right)\right) u_{1}^{*}-A_{2}\left(x_{1}^{*}\right) u_{2}-\ldots-A_{2 n-1}\left(x_{1}^{*}\right) u_{2 n-1}^{*}-A_{2 n}\left(x_{1}^{*}\right) u_{2 n}- \\
& -B_{1}\left(x_{1}^{*}\right) u_{1}^{* *}-B_{2}\left(x_{1}^{*}\right) u_{3}^{* *}-B_{3}\left(x_{1}^{*}\right) u_{5}^{* *}-\ldots-B_{n}\left(x_{1}^{*}\right) u_{2 n-1}^{* *}=f\left(x_{1}^{*}\right) \\
& -A_{0}\left(x_{2}\right) u_{0}-A_{1}\left(x_{2}\right) u_{1}^{*}+\left(1-A_{2}\left(x_{2}\right)\right) u_{2}-\ldots-A_{2 n-1}\left(x_{2}\right) u_{2 n-1}^{*}-A_{2 n}\left(x_{2}\right) u_{2 n}- \\
& -B_{1}\left(x_{2}\right) u_{1}^{* *}-B_{2}\left(x_{2}\right) u_{3}^{* *}-B_{3}\left(x_{2}\right) u_{5}^{* *}-\ldots-B_{n}\left(x_{2}\right) u_{2 n-1}^{* *}=f\left(x_{2}\right)
\end{aligned}
$$




$$
\begin{aligned}
& -A_{0}\left(x_{2 n-1}{ }^{*}\right) u_{0}-A_{1}\left(x_{2 n-1}{ }^{*}\right) u_{1}^{*}-A_{2}\left(x_{2 n-1}{ }^{*}\right) u_{2}-\ldots+\left(1-A_{2 n-1}\left(x_{2 n-1}{ }^{*}\right)\right) u_{2 n-1}{ }^{*}-A_{2 n}\left(x_{2 n-1}{ }^{*}\right) u_{2 n}- \\
& -B_{1}\left(x_{2 n-1}^{*}\right) u_{1}^{* *}-B_{2}\left(x_{2 n-1}^{*}\right) u_{3}^{* *}-B_{3}\left(x_{2 n-1}{ }^{*}\right) u_{5}^{* *}-\ldots-B_{n}\left(x_{2 n-1}{ }^{*}\right) u_{2 n-1}{ }^{* *}=f\left(x_{2 n-1}{ }^{*}\right) \\
& -A_{0}\left(x_{2 n}\right) u_{0}-A_{1}\left(x_{2 n}\right) u_{1}^{*}-A_{2}\left(x_{2 n}\right) u_{2}-\ldots-A_{2 n-1}\left(x_{2 n}\right) u_{2 n-1}^{*}+\left(1-A_{2 n}\left(x_{2 n}\right)\right) u_{2 n}- \\
& -B_{1}\left(x_{2 n}\right) u_{1}^{* *}-B_{2}\left(x_{2 n}\right) u_{3}^{* * *}-B_{3}\left(x_{2 n}\right) u_{5}^{* *}-\ldots-B_{n}\left(x_{2 n}\right) u_{2 n-1}{ }^{* *}=f\left(x_{2 n}\right) \\
& -A_{0}\left(x_{1}^{* * *}\right) u_{0}-A_{1}\left(x_{1}^{* *}\right) u_{1}^{*}-A_{2}\left(x_{1}^{* * *}\right) u_{2}-\ldots-A_{2 n-1}\left(x_{1}^{* *}\right) u_{2 n-1}{ }^{* *}+A_{2 n}\left(x_{1}^{* *}\right) u_{2 n}+ \\
& +\left(1-B_{1}\left(x_{1}^{* *}\right)\right) u_{1}^{* *}-B_{2}\left(x_{1}^{* *}\right) u_{3}^{* *}-B_{3}\left(x_{1}^{* *}\right) u_{5}^{* *}-\ldots-B_{n}\left(x_{1}^{* *}\right) u_{2 n-1}^{* * *}=f\left(x_{1}^{* * *}\right) \\
& -A_{0}\left(x_{3}^{* *}\right) u_{0}-A_{1}\left(x_{3}^{* *}\right) u_{1}^{*}-A_{2}\left(x_{3}^{* *}\right) u_{2}-\ldots-A_{2 n-1}\left(x_{3}^{* *}\right) u_{2 n-1}^{* *}-A_{2 n}\left(x_{3}^{* *}\right) u_{2 n}- \\
& -B_{1}\left(x_{3}^{* *}\right) u_{1}^{* *}+\left(1-B_{2}\left(x_{3}^{* *}\right)\right) u_{3}^{* *}-B_{3}\left(x_{3}^{* *}\right) u_{5}^{* *}-\ldots-B_{n}\left(x_{3}^{* *}\right) u_{2 n-1}^{* *}=f\left(x_{3}^{* *}\right) \ldots \\
& -A_{0}\left(x_{2 n-1}{ }^{* *}\right) u_{0}-A_{1}\left(x_{2 n-1}{ }^{* *}\right) u_{1}{ }^{*}-A_{2}\left(x_{2 n-1}{ }^{* *}\right) u_{2}-\ldots-A_{2 n-1}\left(x_{2 n-1}{ }^{* *}\right) u_{2 n-1}{ }^{*}-A_{2 n}\left(x_{2 n-1}{ }^{* *}\right) u_{2 n}- \\
& -B_{1}\left(x_{2 n-1}{ }^{* *}\right) u_{1}^{* *}-B_{2}\left(x_{2 n-1}^{* *}\right) u_{3}^{* *}-B_{3}\left(x_{2 n-1}^{* *}\right) u_{5}^{* *}-\ldots+\left(1-B_{n}\left(x_{2 n-1}^{* *}\right)\right) u_{2 n-1}^{* *}=f\left(x_{2 n-1}^{* *}\right)
\end{aligned}
$$

where

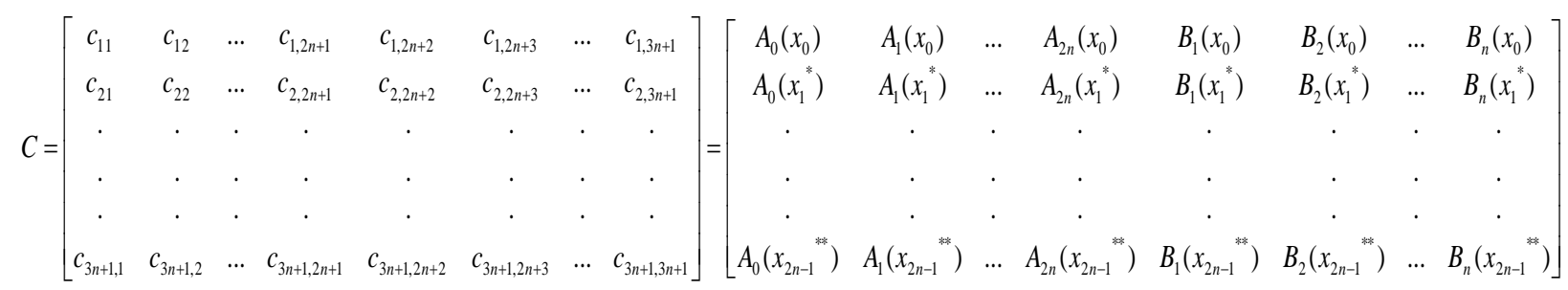

and if we write,

$$
\begin{aligned}
& Z=\left[u_{0}, u_{1}^{*}, u_{2}, \ldots, u_{2 n}, u_{1}^{* *}, u_{3}^{* *}, \ldots, u_{2 n-1}^{* *}\right]^{T} \\
& D=\left[f\left(x_{0}\right), f\left(x_{1}^{*}\right), f\left(x_{2}\right), \ldots, f\left(x_{2 n}\right), f\left(x_{1}^{* *}\right), f\left(x_{3}^{* *}\right), \ldots, f\left(x_{2 l-1}^{* *}\right)\right]^{T}
\end{aligned}
$$

and the identity matrix $E_{3 n+1,3 n+1}$, then we can rewrite the above system of equations (*) as matrix form

$$
\left(E_{3 n+1,3 n+1}-C\right) Z=D
$$

\section{ILLUSTRATIVE EXAMPLE}

Let's consider the following LFSIESK,

$$
u(x)=\int_{0}^{1}(1+\sqrt{x} \sqrt{s}) u(s) d(\sqrt{s})-\frac{1}{2} \sqrt{x}, \quad x \in[0,1]
$$

Where

$$
\begin{aligned}
& K(x, s)=1+\sqrt{x} \sqrt{s}, \quad \phi(x)=\sqrt{x}, \quad \psi(x)=0, \quad f(x)=-\frac{1}{2} \sqrt{x}, \quad x_{i}=a+i h \\
& h=\frac{b-a}{2 n}=\frac{1-0}{2.5}=\frac{1}{10}=0.1, \quad x_{0}=0, x_{2}=0.2, x_{4}=0.4, x_{6}=0.6, x_{8}=0.8, x_{10}=1
\end{aligned}
$$

\begin{tabular}{|c|c|c|}
\hline $\mathrm{n}$ & GTM & GSM \\
\hline 5 & 0.3117197700 & 0.2889598762 \\
\hline 10 & 0.3307884409 & 0.2988484072 \\
\hline
\end{tabular}




\section{CONCLUSION}

The Linear Fredholm-Stieltjes Integral Equations of Second Kind are usually difficult to solve analytically. In many cases, it is required to find the approximate solutions, for this aim the presented methods can be proposed. From numerical examples, it can be seen that the proposed numerical methods are efficient and accurate to estimate the solution of these equations, also, it can be seen that when the values $\mathrm{h}$ decreases, the absolute errors decrease to small values.

\section{REFERENCES}

[1] D. Baleanu, K. Diethelm, E. Scalas, and J. J. Trujillo, Fractional Calculus: Models and Numerical Methods, World Scientific, NewYork, USA, 2012.

[2] K. Diethelm, The Analysis of Fractional Diffrential Equations,LectureNotesinMathematics,Springer,Berlin,Germany,2010

[3] R. Hilfer, Applications of Fractional Calculus in Physics, WorldScientifi, River Edge, NJ, USA, 2000.

[4] V. E. Tarasov, Fractional Dynamics: Applications of Fractional Calculus to Dynamics of Particles, Fields and Media, Nonlinear Physical Science, Springer, Heidelberg, Germany; Higher Education Press,Beijing,China,2010.

[5] N. T. Dung, "Fractional stochastic diffrential equations with applications to fiance," Journal of Mathematical Analysis and Applications, vol. 397, no. 1, pp. 334-348, 2013

[6] A. M. Wazwaz ,Linear and Nonlinear Integral Equations, Methods and Applications, Springer, Higher Education Press, Beijing and Springer-Verlag, Berlin, 2011

[7] M. Federson, J.G. Mesquita, A.Slavik, Basic results for functional diff erential and dynamic equations involving impulses, Mathematis cheNachrichten, Volume 286, Issue 2-3, 181-204, 2013

[8] M. Federsonand R. Bianconi, Linear Fredholm integral equations concerning the integral of Kurzweil, J. Appl. Anal., 8 (1), 81-108, 2002.

[9] M. Federson and R. Bianconi, Existence and uniqueness of solutions for linear Fredholm- Stieltjes integral equations via Henstock-Kurzweil integral, Integration: Mathematical Theory and Applications, accepted.

[10] L.J. Paradiso, Solutions of Bounded Variation of the Fredholm- Stieltjes Integral Equation, Presented to the Society, 1932

[11] S. Schwabik, On Fredholm- Stieltjes Integral Equations(Preliminary communication), Commentationes Mathematicae Universitatis Carolinae, Vol. 12 (1971), No. 4,845-849,1971

[12] L.M. Delves, J.L. Mohamed, Computational methods for integral equations, Cambridge University Press, New York,USA, 1985

[13] H. Wolfgang, Integral Equations; theory and numerical treatment, Birkhauser, Basel,Germany, 1995

[14] A. Asanov, M.H. Chelik, A. Chalish, Approximating the Stieltjes Integral Using the Generalized Trapezoid Rule, Le Matematiche, Vol.L XVI- Fasc.II,Pp.13-21, 2011

[15] A. Asanov, M.H. Chelik, M. Abdujabbarov, Approximating the Stieltjes Integral Using the Generalized Midpoint Rule,MATEMATIKA, Vol. 27, Num. 2, 139-148, 2011

[16] A. Asanov, M.H. Chelik, M. Sezer, Approximating the Stieltjes Integral Using the Generalized Simpson's Rule, Communications in Differential and Difference Equations, Volume 3, Number 1, 1-11, 2012

[17] I.P.Natanson, Theory of functions with the real variable, Nauka, Moscow (in Russian), 1974 